\title{
ON THE EXISTENCE AND BOUNDEDNESS OF SOLUTIONS OF NONLINEAR PARTIAL DIFFERENTIAL EQUATIONS OF PARABOLIC TYPE
}

\author{
BY \\ RICHARD BELLMAN( ${ }^{(1)}$
}

1. Introduction. We intend to investigate in this paper the existence and boundedness of solutions of nonlinear partial differential equations of the type occurring in the mathematical theory of heat conduction. In three dimensions, the equation has the form

$$
u_{x x}+u_{y y}+u_{z z}-q u_{t}=F(u, x, y, z, t), \quad t>0,
$$

where $q$ is a positive constant which we may, without loss of generality, take to be 1 , and $F(u, x, y, z, t)$ is a nonlinear function of $u$. The boundary conditions will be of the following type:

$$
\begin{array}{rlr}
u & =0 \text { on } B, & t>0, \\
\lim _{t \rightarrow 0} u & =f(x, y, z), & x, y, z \text { inside } R,
\end{array}
$$

where $B$ is the boundary of a closed convex region, $R$, in ordinary Euclidean 3 -space, and $f(x, y, z)$ is defined over $R$. The variable $t$ will range over the interval $(0, \infty)$.

If we take the surface $B$ to be the boundary of a cube, and impose the conditions corresponding to (1.2),

$$
\begin{aligned}
u & =0 \text { for } x=0, \pi, \text { and all } y, z, \\
u & =0 \text { for } y=0, \pi, \text { and all } x, z, \\
u & =0 \text { for } z=0, \pi, \text { and all } x, y, \quad t>0, \\
\lim _{t \rightarrow 0} u & =f(x, y, z),
\end{aligned}
$$

the problem is connected with the subject of heat conduction in a crystal.

The main part of the paper will be devoted to the equation (1.1) and the boundary conditions (1.3). The methods developed for this specific purpose will then be discussed with reference to other applications.

The question of existence and uniqueness of functions satisfying (1.1) and the boundary conditions $(1.3)$ has been treated by Siddiqi $[10,11,12]\left({ }^{2}\right)$ for the case where $F(u, x, y, z, t) \equiv u^{2}$, and by Minakshisundaram [7] for the gen-

Presented to the Society, February 28, 1948; received by the editors February 14, 1947.

(1) The results of this paper were obtained while engaged in research sponsored by the Office of Naval Research.

(2) Numbers in brackets refer to the references cited at the end of the paper. 
eral equation of Sturm-Liouville type

$$
u_{x x}+u_{y y}+u_{z z}-u_{t}=q(x, y, z) u+F(u, x, y, z, t), \quad t>0 .
$$

Solutions of nonlinear partial differential equations of parabolic type have been discussed in two classic papers by Gevrey [4]( $\left.{ }^{3}\right)$ by methods quite different from those used here. His methods apply to various types of finite regions in $(x, y, z, t)$ space, and not to the type of region treated here.

As stated by Siddiqi, his methods apply also to the more general situation where $F=\sum_{n=z}^{\infty} p_{n}(x, y, z) u^{n}$, where the $p_{n}$ are uniformly bounded functions of $x, y, z$, and $t$, with uniformly bounded second derivatives. As we shall see subsequently, the boundedness condition with respect to $t$ can be eased quite a bit.

The method of proof used by $\operatorname{Siddiqi}\left({ }^{4}\right)$ requires the solution, by means of successive approximations, of an infinite system of nonlinear integral equations. The method imposes stringent restrictions upon $f(x, y, z)$, and thus both weakens the theorem and lessens its range of applicability. One object of this paper is to lighten these restrictions to a considerable degree.

The main purpose of the paper is, however, to demonstrate a general method of attacking problems connected with equations such as (1.1). The method is similar in principle to that used by the author [1] to treat ordinary nonlinear differential equations. Although the main feature of the method is classical-namely the conversion of the differential equation into a suitable integral equation - the application of a result from the theory of multiple Fourier series seems novel, and is of material assistance in carrying through the proof. Before continuing in this direction, we shall discuss some general concepts.

It seems appropriate in treating nonlinear problems to introduce the concept of stability of solutions of partial differential equations. The definition may be generalized quite easily from the usual one pertaining to systems of ordinary differential equations. Let us first, for the sake of comparison, consider the definition of Liapounoff-Poincaré stability. If we have a system of differential equations

$$
\frac{d y_{i}}{d t}=\sum_{j=1}^{N} a_{i j}(t) y+f_{i}\left(y_{1}, y_{2}, \cdots, y_{N}, t\right), \quad i=1,2, \cdots, N, t \geqq 0,
$$

where the $f_{i}$ represent nonlinear terms, and

$$
f_{i}(0,0, \cdots, 0, t)=0, \quad i=1,2, \cdots, N,
$$

a solution is $y_{i}=0, i=1,2, \cdots, N$. This solution is said to be stable in the

(3) Reference to the other paper is given here.

(4) Minakshisundaram's methods are similar to those of Siddiqi, who in turn used methods hased upon those of L. Lichtenstein. 
sense of Liapounoff-Poincaré, if every solution, $Y=\left(Y_{i}\right)$, of $(1.5)$, with the initial conditions

$$
Y_{i}(0)=c_{i}, \quad i=1,2, \cdots, N
$$

satisfies the inequality

$$
\|Y\|=\sum_{i=1}^{N}\left|Y_{i}(t)\right| \leqq \epsilon, \quad 0 \leqq t<\infty
$$

whenever

$$
\sum_{i=1}^{N}\left|c_{i}\right| \leqq \delta=\delta(\epsilon)
$$

The symbol $\|Y\|$ can be considered as the norm of the solution $Y$. It satisfies the usual requirements for a norm.

With the above as a guide, we can define stability as follows:

Definition. $A$ solution, $u$, of a partial differential equation is said to be stable, if any solution, $U$, of the equation whose boundary values are sufficiently "close" to the boundary values of $u$, remains "close" to $u$ for all values of the independent variables. The term "close" is to be defined by an appropriate metric.

As we see immediately from the definition, the idea of stability is associated with the very interesting questions as to the dependence, continuous or not, of the solutions of differential equations upon the boundary conditions. For ordinary nonlinear differential equations this dependence is complicated by the existence of movable singularities.

A solution $u$ is said to be relatively stable with respect to a class of functions, $S$, if the above definition is taken only to refer to solutions, $U$, belonging to $S$. Since the question of uniqueness of solutions of partial differential equations is in general very difficult, we are forced to consider relative stability.

Of course, unless one can show that many solutions of the particular partial differential equation exist, the definition is vacuous. It is entirely possible to construct a partial differential equation for which $u=0$ would be an isolated solution.

In the present situation, we shall consider the class, $S$, of possible solutions of (1.1) to be those functions satisfying the first boundary condition of (1.3), plus conditions we shall specify later. Thus the only variation in the boundary values shall be in the function $f(x, y, z)$. If $F(u, x, y, z, t)=0, u=0$ is a solution of (1.1) and (1.3), if $f(x, y, z)=0$. To show that $u=0$ is stable relative to $S$, we shall have to show that any solution of (1.1) and (1.3) belonging to $S$ remains small for $x, y, z$ in $R$ and $0 \leqq t<\infty$, provided that $f(x, y, z)$ as measured by some norm is sufficiently small, in the general case $F \neq 0$.

Siddiqi expanded $f(x, y, z)$ in its Fourier sine series over the cube $R$. 


$$
f(x, y, z) \sim \sum_{l, m, n=1}^{\infty} a_{l m n} \sin l x \sin m y \sin n z
$$

(here and in what follows, we shall use the symbol $\sim$ to mean "has the Fourier series expansion, or, more generally, the orthogonal series expansion"), and defined

$$
\text { Norm } f=\|f\|=\sum_{l, m, n=1}^{\infty}\left|a_{l m n}\right| .
$$

As may be seen by computing the coefficients, this attaches the norm $+\infty$ to the function 1, which shows that this definition of norm is not very satisfactory.

A more natural norm, analogous to that defined by (1.8), would be

$$
\|f\|=\max _{R}|f(x, y, z)|,
$$

where $R$ is the region under consideration, in our case a cube.

Since the problem is essentially of physical origin, we shall consider only functions, $f(x, y, z)$, which are continuous over $R$. We point out, however, that as far as the mathematical treatment is concerned, we could use the norm

$$
\|f\|=\text { essential upper bound } f(x, y, z) \text {, }
$$

and ask only that $\lim _{t \rightarrow 0} u=f(x, y, z)$ hold almost everywhere.

Let $R \times T$ represent the 4-dimensional topological product of $R$ and the infinite interval, $0 \leqq t<\infty$. For a function, $u$, of the four variables, $x, y, z, t$, we define similarly

$$
\|u\|=\max _{R \times T}|u|
$$

We shall use the same notation, "\| $\|$," for both norms, since it will be clear from the character of the function which of the two is meant.

We now precise the class $S$. In addition to satisfying the first boundary condition of (1.3), we require that $u$ possess continuous second partial derivatives, $u_{x x}, u_{y y}, u_{z z}$, for any $t>0$, and that the convergence of $u(x, y, z, t)$ to $f(x, y, z)$ is such that $|u(x, y, z, t)| \leqq \phi(x, y, z)$, as $t \rightarrow 0$, and $\int_{R} \phi(x, y, z) d V$ exist. This condition is introduced to permit us to use various interchanges of the limit process and integration. Again, if we were interested in an almostall theory, we could replace continuous by Lebesgue-integrable.

Before stating our main result we want to mention one other point. For an ordinary system of differential equations of the $n$th order, as defined by (1.5), the general solution will depend upon $N$ parameters, and thus we may 
speak of an $N$-dimensional family of solutions, or an $N$-dimensional manifold of solutions.

The solutions of a partial differential equation, on the other hand, usually depends upon a set of arbitrary functions, and thus may be considered to depend upon an enumerable, or non-enumerable, number of parameters, depending upon how one regards a function. An alternative point of view consists of regarding the solution as a functional. Thus an $N$-dimensional functional manifold of solutions is defined as one depending upon $N$ functions. We shall use this terminology in stating our principal result.

THEOREM 1. Consider the equation

$$
u_{x x}+u_{y y}+u_{z z}-u_{t}=F(u, x, y, z, t)(5)
$$

with the boundary conditions

$$
\begin{aligned}
& u=0 \text { for } x=0, \pi, \text { and all } y, z, \\
& u=0 \text { for } y=0, \pi, \text { and all } x, z, \\
& u=0 \text { for } z=0, \pi, \text { and all } x, y,
\end{aligned}
$$

(2) $\quad \lim _{t \rightarrow 0} u=f(x, y, z)$,

$x, y, z$ inside $R$.

Let

(1) $f(x, y, z)$ be a continuous function over $R$, and $\|f\| \leqq c_{1}$, where $c_{1}$ is a small constant to be determined,

(2) $F(u, x, y, z, t)=\sum_{n=2}^{\infty} d_{n} u^{n}, \quad \sum_{n=2}^{\infty}\left\|d_{n}\right\||u|^{n}<\infty$,

$$
\text { for }|u| \leqq \rho>c_{1}>0 .
$$

Under these conditions, there exists a solution which is uniformly bounded in $R \times T$, and approaches 0 as $t \rightarrow \infty$.

This solution is the only solution belonging to $S$.

Furthermore, this solution is an element of a one-dimensional functional manifold of solutions of (1.14), satisfying (1.15) (1).

As mentioned above, the proof depends upon the conversion of the partial differential equation into an appropriate integral equation. The method of successive approximations is then used to obtain a solution of the integral equation. To apply the method effectively a lemma from the theory of multiple Fourier series due to Eversull [2] is used.

(5) With slight additional complications we could handle the case where $F=F\left(u, u_{x}, u_{y}, u_{z}\right.$, $\left.u_{t}, x, y, z, t\right)$, the partial derivatives entering nonlinearly. One method of doing this is to assume that $f(x, y, z)$ has continuous partial derivatives $f_{x}, f_{y}, f_{z}$ over $R$, which have sufficiently small norms. 
Application of this method to other types of partial differential equations such as the Sturm-Liouville equation, (1.4), and the equation

$$
u_{x x x x}+u_{y y y y}+u_{z z z z}-u_{t}=F(u, x, y, z, t)
$$

are also discussed, as well as to other types of regions such as spheres and infinite cylinders.

In treating (1.4) we shall use results of Haar concerning the equi-convergence and equi-summability of the expansion of functions in the eigen-functions of a Sturm-Liouville equation with the Fourier sine expansion of the function.

Finally, as a further application of the method, we shall discuss a type of nonlinear integro-differential equation.

2. Preliminary results and lemmas. In this section we shall discuss some results required for the proof of Theorem 1.

We begin with the following result due to Eversull which is fundamental in our proof.

Lemma 1 (Eversull). Let $f(x, y, z)$ be continuous over the closed region, defined by $-\pi \leqq x, y, z \leqq \pi$, and let

$$
\begin{aligned}
f(x, y, z) & \sim \sum_{l, m, n=-\infty}^{\infty} a_{l m n} e^{i(l x+m y+n z)}, \\
\phi(x, y, z, t) & =\sum_{l, m, n=-\infty}^{\infty} a_{l m n} e^{i(l x+m y+n z)-\left(l^{2}+m^{2}+n^{2}\right) t}, \quad t>0 .
\end{aligned}
$$

Then

$$
\|\phi\| \leqq d_{1}\|f\|,
$$

where $d_{1}$ is a constant, independent of $x, y, z, t$ and $f(x, y, z)$.

The norms are those defined by (1.13) and (1.11) respectively.

Proof. We refer to Eversull [2] for the proof which is obtained by using Fejer's theorem on the convergence of $(C, 1,1,1)$ means of triple Fourier series. We have phrased the lemma in terms of the intervals $(-\pi, \pi)$, since it will be convenient to regard some of the functions occurring later, defined only in intervals $(0, \pi)$, as odd functions, defined by this property over the interval $(-\pi, 0)$. We have used the exponential form $e^{i(l x+m y+n z)}$ to avoid writing out all the various combinations such as $\sin l x \cos m y \cos n z$.

Eversull also proves that

$$
\lim _{t \rightarrow 0} \phi(x, y, z, t)=f(x, y, z)
$$

for all values of $x, y, z$, belong to $R$.

LEMMA 2. Every solution of (1.14) which belongs to $S$ satisfies the nonlinear 
integral equation

$$
\begin{array}{rl}
u & u \\
= & u_{0}-\int_{0}^{t}\left\{\sum_{l, m, n=1}^{\infty} F_{l m n}\left(u\left(t_{1}\right), t_{1}\right) e^{-\left(l^{2}+m^{2}+n^{2}\right)\left(t-t_{1}\right)} \sin l x \sin m y \sin n z\right\} d t_{1} \\
& =u_{0}-T(u)
\end{array}
$$

where

$$
F(u, x, y, z, t) \sim \sum_{l, m, n=1}^{\infty} F_{l m n}(u, t) \sin l x \sin m y \sin n z\left(^{6}\right),
$$

and $u_{0}$ is defined below.

Proof. We begin with the equation

$$
u_{x x}+u_{y y}+u_{z z}-u_{t}=F(u) .
$$

We shall use the following abbreviations throughout the remainder of the paper:

$$
\begin{aligned}
\psi_{l m n} & =\sin l x \sin m y \sin n z & \\
u_{0} & =\sum_{l, m, n=1}^{\infty} a_{l m n} \psi_{l m n} e^{-\left(l^{2}+m^{2}+n^{2}\right) t}, & t>0 .
\end{aligned}
$$

Multiply (2.7) through by $\psi_{l m n}$ and integrate over $R$. If we use integration by parts, and the boundary conditions, the left-hand side becomes

$$
-\left(l^{2}+m^{2}+n^{2}\right) u_{l m n}-d u_{l m n} / d t,
$$

and thus from (2.7) we obtain

$$
-\left(l^{2}+m^{2}+n^{2}\right) u_{l m n}-d u_{l m n} / d t=F_{l m n},
$$

where $u_{l m n}$ is the Fourier coefficient of $u$, and $F_{l_{m n}}$ the Fourier coefficient of $F$. We have used the interchange of $\partial / \partial t$ and integration over $R$ which is valid, provided $\partial u / \partial t$ is $L$-integrable over $R$ for any $t>0$. This follows from Fubini's theorem.

We now use the condition that $|u| \leqq \phi(x, y, z)\left({ }^{7}\right)$ and $\int_{R} \phi d V<\infty$ to deduce that

$$
\lim _{l \rightarrow 0} u_{l m n}=\lim _{t \rightarrow 0}\left(\frac{2}{\pi}\right)^{3} \int_{R} u \psi_{l m n} d V=\left(\frac{2}{\pi}\right)^{3} \int_{R} f \psi_{l m n} d V=a_{l m n} .
$$

Thus the solution of $(2.10)$ is given by

(6) $F_{l m n}(u, t)=F_{l m n}(u(t), t)$, where it is understood that the other variables $x, y, z$ are also present in $u$; also $F(u)$ will be used as a substitute for $F(u, x, y, z, t)$.

( 7$)$ Defined in the second paragraph below (1.13). 


$$
u_{l m n}=a_{l m n} e^{-\left(l^{2}+m^{2}+n^{2}\right) t}-\int_{0}^{t} e^{-\left(l^{2}+m^{2}+n^{2}\right)\left(t-t_{1}\right)} F_{l m n}\left(t_{1}\right) d t_{1} .
$$

Whence, since

$$
u \sim \sum_{l, m, n=1}^{\infty} u_{l m n} \psi_{l m n}
$$

we have

$$
\begin{aligned}
u \sim \sum_{l, m, n=1}^{\infty}\left[a_{l m n} \psi_{l m n} e^{-\left(l^{2}+m^{2}+n^{2}\right) t}\right] & \\
& \quad-\sum_{l, m, n=1}^{\infty}\left[\psi_{l m n} \int_{0}^{t} e^{-\left(l^{2}+m^{2}+n^{2}\right)\left(t-t_{1}\right)} F_{l m n}\left(t_{1}\right) d t_{1}\right] .
\end{aligned}
$$

Since we know, to begin with, that $u$ has a uniformly convergent sine expansion for any $x, y, z$, inside $R$, and $t>0$, since $u_{x x}, u_{y y}, u_{z z}$ exist and are $L$-integrable, we can write equality in (2.14). We note, incidentally, that we have proved a uniqueness theorem for the ordinary, linear partial differential equation where $F(u) \equiv 0$.

We now wish to show that we can interchange summation and integration, and write this relation as

$$
u=u_{0}-\int_{0}^{t}\left\{\sum_{l, m, n=1}^{\infty} F_{l m n}\left(t_{1}\right) e^{-\left(l^{2}+m^{2}+n^{2}\right)\left(t-t_{1}\right)} \psi_{l m n}\right\} d t_{1} .
$$

We may note that an application of Lemma 1 shows that the integrand is bounded by $d_{1} \max _{R \times(0, t)}|F(u)|$, and thus integrable. The factors $e^{-\left(l^{2}+m^{2}+n^{2}\right)\left(t-t_{1}\right)}$ ensure absolute convergence in the half-open interval $0 \leqq t_{1}<t$, and we may define the function at $t=t_{1}$, by continuity, to be $F(u)$. To show the legitimacy of interchange of limits, we break up the interval $(0, t)$ into two intervals $(0, t / 2)$ and $(t / 2, t)$. In $(0, t / 2)$, the integrand is the sum of an absolutely convergent series and thus uniformly convergent. In the interval $(t / 2, t)$, the function $F(u)$ has a uniformly convergent Fourier series since all partial derivatives $F(u)_{x x}, F(u)_{y y}, F(u)_{z z}$ exist, and thus the interchange is permissible, since the factors $e^{-\left(l^{2}+m^{2}+n^{2}\right)\left(t-t_{1}\right)}$ will only aid and abet the uniform convergence. When we say uniform convergence, we mean uniform with respect to $t$, as well as $x, y, z$. As far as the interchange of limits is concerned, it is only the uniformity with respect to $t$ that matters.

We shall use this method of subdividing the interval of integration several times in the course of the proof.

If we do not wish to rely upon any results from the theory of multiple Fourier series, apart from Lemma 1, we can use a simple argument based upon the continuity of $u$ as a function of $t$. We shall require a proof of this sort to 
treat the general stability theorem we shall present later, and therefore we shall give it now.

Break up the interval $(0, t)$ into the intervals $(0, t-d),(t-d, t)$, where $0<d<t$. In the interval $(0, t-d)$, we have absolute, and hence uniform, convergence because the terms are majorized by const. $X e^{-\left(l^{2}+m^{2}+n^{2}\right) d}$.

Also, as mentioned above, the integral

$$
I=\int_{0}^{t}\left\{\sum_{l, m, n} F_{l m n} e^{-\left(l^{2}+m^{2}+n^{2}\right)\left(t-t_{1}\right)} \psi_{l m n}\right\} d t_{1}
$$

exists, since the integrand is uniformly bounded by $d_{1}\|F(u)\|$ for every $t_{1}$. The series in the integrand converges uniformly in any half-open interval $0 \leqq t_{1}<t$.

The difference between $I$ and the infinite series occurring in (2.14) is given by

$$
\int_{t-d}^{t} \sum_{l, m, n}-\sum_{l, m, n} \int_{t-d}^{t}
$$

since the interchange of summation and integration in the interval $(0, t-d)$ permits cancellation of the common part. Now as $d \rightarrow 0$

$$
\left|\int_{t-d}^{t} \sum_{l, m, n} d t_{1}\right| \leqq d d_{1} \max _{t-d \leqq t_{1} \leqq t}|F(u)| \rightarrow 0 \quad \text { as } \quad d \rightarrow 0 .
$$

On the other hand, the second term

$$
\sum_{l, m, n} \int_{t-d}^{t}
$$

is precisely $u(t)-u(t-d)$. Since $u$ is a solution of the partial differential equation and the boundary conditions, $u$ is a continuous function of $t$ and thus $u(t)-u(t-d) \rightarrow 0$ as $d \rightarrow 0$. Thus (2.15) is valid.

3. Proof of stability. Before proceeding to the main theorem, we want to show that the solution $u=0$ of (1.4) is stable relative to the class $S$. To obtain this result we require much less of $F(u)$ than Theorem 1 does. We shall prove the following:

THEOREM 2. Consider the equation

$$
u_{x x}+u_{y y}+u_{z z}-u_{t}=F(u, x, y, z, t)
$$

with the boundary conditions of (1.2). Let

$$
F(u, x, y, z, t)=o(u)
$$$$
\text { as } u \rightarrow 0 \text {, uniformly in } x, y, z \text {. }
$$

Then $u$ is stable relative to the class $S$.

Proof. Using Lemma 2, we know that every solution belonging to $S$ satis- 
fies the integral equation

$$
u=u_{0}-\int_{0}^{t}\left\{\sum_{l, m, n=1}^{\infty} F_{l m n}\left(t_{1}\right) e^{-\left(l^{2}+m^{2}+n^{2}\right)\left(t-t_{1}\right)} \psi_{l m n}\right\} d t_{1} .
$$

From Lemma 1 it follows that

$$
\left\|u_{0}\right\| \leqq d_{1}\|f\| .
$$

We shall have shown the required stability it we prove that $\|u\|<2 d_{1}\|f\|$, for small enough $\|f\|$.

The method used is an adaptation of a method used by Levinson for ordinary differential equations.

Choose a $d>0$, which will be determined more precisely below. Let $t_{1}$ be the first value of $t>0$, for which $\max _{R}|u|=2 d_{1}\|f\|$, and assume to begin with that $t_{1}>d$. The case $0<t_{1} \leqq d$ is simpler. Then from (3.3) it follows that, for $t=t_{1}$,

$$
|u| \leqq\left\|u_{0}\right\|+\left|\int_{0}^{t_{1}-d}\right|+\left|\int_{t_{1}-d}^{t_{1}}\right| .
$$

In the interval $\left(0, t_{1}-d\right)$, each Fourier coefficient satisfies the inequality

$$
\left|F_{l m n}(t)\right| \leqq \max _{R \times\left(0, t_{1}\right)}|F(u)|
$$

and thus the integrand is majorized by

$$
\max _{R \times\left(0, t_{1}\right)}|F(u)| \sum_{l, m, n=1}^{\infty} e^{-\left(l^{2}+m^{2}+n^{2}\right)\left(t-t_{1}\right)} .
$$

Hence

$$
\begin{aligned}
\left|\int_{0}^{t_{1}-d}\right| & \leqq \max _{R \times\left(0, t_{1}\right)}|F(u)| \sum_{l, m, n=1}^{\infty} \frac{e^{-\left(l^{2}+m^{2}+n^{2}\right) d}-e^{-\left(l^{2}+m^{2}+n^{2}\right) t}}{l^{2}+m^{2}+n^{2}} \\
& \leqq \max _{R \times\left(0, t_{1}\right)}|F(u)| \sum_{l, m, n=1}^{\infty} \frac{e^{-\left(l^{2}+m^{2}+n^{2}\right) d}}{l^{2}+m^{2}+n^{2}} .
\end{aligned}
$$

In $\left(t_{1}-d, t_{1}\right)$, the integrand is majorized by $d_{1} \max _{R \times\left(0, t_{1}\right)}|F(u)|$, if we use Lemma 1, and thus

$$
\left|\int_{t-d}^{t_{1}}\right| \leqq d d_{1} \max _{R \times\left(0, t_{1}\right)}|F(u)| .
$$

Collecting these results, we obtain

$$
\left|u\left(t_{1}\right)\right| \leqq\left\|u_{0}\right\|+\max _{R \times\left(0, l_{1}\right)}|F(u)|\left[\sum_{l, m, n=1}^{\infty} \frac{e^{-\left(l^{2}+m^{2}+n^{2}\right) d}}{l^{2}+m^{2}+n^{2}}+d d_{1}\right] .
$$


Choose $d$, for simplicity, so that the bracketed term on the right-hand side reduces to $2 d d_{1}$. Then

$$
\max _{R}\left|u\left(t_{1}\right)\right| \leqq\left\|u_{0}\right\|+2 d d_{1} \max _{R \times\left(0, t_{1}\right)}|F(u)| .
$$

Since $|F(u)|=o(|u|)$ as $u \rightarrow 0$, we see that we can choose $\|f\|$ small enough so that, knowing $\max _{R \times\left(0, t_{1}\right)}\left|u\left(t_{1}\right)\right| \leqq 2 d_{1}\|f\|$, we have

$$
\max _{R}\left|u\left(t_{1}\right)\right| \leqq d_{1}\|f\|+2 d d_{1}\left(o\left(\max _{R \times\left(0, t_{1}\right)}|u|\right)\right)<2 d_{1}\|f\|,
$$

and thus a contradiction of our assumption concerning the point $t_{1}$.

If $0<t_{1} \leqq d$, we have

$$
u \leqq\left\|u_{0}\right\|+\left|\int_{0}^{t_{1}}\right| \leqq\left\|u_{0}\right\|+d d_{1} \max _{R \times\left(0, t_{1}\right)}|F(u)|,
$$

and the proof continues as above.

The requirement imposed upon $F(u)$ is certainly satisfied if $F(u)$ is a power series of the form $\sum_{n=2}^{\infty} a_{n}(x, y, z) u^{n}$, where the $a_{n}(x, y, z)$ are bounded by $A_{n}$ and $\sum_{n=2}^{\infty} A_{n} u^{n}<\infty$. However, more generally, we can take $F(u)$ to be $|u|^{3 / 2}$ or $u(\log |u|)^{-1}$, and so on.

It should be mentioned that stability depends upon the norm used to measure "closeness." The solution $u=0$ would not be stable under the condition of Theorem 1 , if we required that

$$
\max _{R \times T}\left|u_{x x}+u_{y y}+u_{z z}\right|
$$

be uniformly small.

It also seems of interest to point out that there may be several stable solutions, depending upon the zeroes of $F(u)$. Thus if $F(u)=u^{2}(u-1)^{2}$, there will be neighborhoods of solutions with norms close to 0 and close to 1 .

4. Proof of Theorem 1. Using Lemma 2, we convert the partial differential equation into the integral equation

$$
\begin{aligned}
u & =u_{0}-\int_{0}^{t}\left\{\sum_{l, m, n=1}^{\infty} F_{l m n}\left(t_{1}\right) e^{-\left(l^{2}+m^{2}+n^{2}\right)\left(t-t_{1}\right)} \psi_{l m n}\right\} d t_{1} \\
& =u_{0}-T(u)
\end{aligned}
$$

We now consider the sequence defined by the successive approximations

$$
\begin{aligned}
u_{0} & =u_{0}, \\
u_{n+1} & =u_{0}-T\left(u_{n}\right), \quad n \geqq 0 .
\end{aligned}
$$

It is clear from what has preceded that each member of the sequence satisfies the boundary conditions. Let us show first that $\left\|u_{n}\right\| \leqq 2 d_{1}\|f\|$, pro- 
vided $\|f\|$ is sufficiently small. The result is true for $n=0$, as a consequence of Lemma 1. We now use induction, assuming that the result holds for $N=0,1,2, \cdots, n$, and proving it for $n+1$.

We shall use the same device used in $\S 3$, breaking up the interval $(0, t)$ into the sub-intervals $(0, t-d),(t-d, t)$ provided $t>d$, and leaving the interval unchanged otherwise. As before, $d$ will be determined in the course of the proof. We have, if $t>d$,

$$
\begin{aligned}
\left\|u_{n+1}\right\| & \leqq\left\|u_{0}\right\|+\left\|\int_{0}^{t-d}\right\|+\left\|\int_{t-d}^{t}\right\| \\
& \leqq d_{1}\|f\|+\left\|F\left(u_{n}\right)\right\|\left[\sum_{l, m, n=1}^{\infty} \frac{e^{-\left(l^{2}+m^{2}+n^{2}\right) d}}{l^{2}+m^{2}+n^{2}}+d d_{1}\right]\left(^{(8)}\right.
\end{aligned}
$$

where we have again used Lemma 1 in the interval $(t-d, t)$. Letting $d$ be chosen so that the coefficient of $\left\|F\left(u_{n}\right)\right\|$ becomes $2 d d_{1}$, we have

$$
\left\|u_{n+1}\right\| \leqq d_{1}\|f\|+2 d d_{1}\left\|F\left(u_{n}\right)\right\| \text {. }
$$

Since for small $\left\|u_{n}\right\|,\left\|F\left(u_{n}\right)\right\| \leqq 2 c_{2}\left\|u_{n}\right\|^{2}$, it is clear that

$$
\left\|u_{n+1}\right\| \leqq d_{1}\|f\|+2 d d_{1}\left(2 c_{2}\right)\left(2 d_{1}\|f\|\right)^{2} \leqq 2 d_{1}\|f\|
$$

if $\|f\|$ is sufficiently small. If $t \leqq d$, we have

$$
\left\|u_{n+1}\right\| \leqq d_{1}\|f\|+d d_{1}\left\|F\left(u_{n}\right)\right\| \leqq 2 d_{1}\|f\| .
$$

The next step is the usual one, proving that $\sum_{n=0}^{\infty}\left(u_{n+1}-u_{n}\right)$ converges uniformly. We have

$$
u_{n+1}-u_{n}=T\left(u_{n}\right)-T\left(u_{n-1}\right) .
$$

$T\left(u_{n}\right)-T\left(u_{n-1}\right)$ will be an integral with limits 0 and $t$ and we break up the interval into the sub-intervals $(0, t-d),(t-d, t)$, if $t>d$, and leave the interval unchanged otherwise. We consider the case $t>d$ first. Then

$$
T\left(u_{n}\right)-T\left(u_{n-1}\right)=\int_{0}^{t-d}+\int_{t-d}^{t} .
$$

In the interval $(0, t-d)$, we use the fact that the Fourier coefficients occurring in the integrand will be the difference of the Fourier coefficients of $F\left(u_{n}\right)$ and $F\left(u_{n-1}\right)$, multiplied by $e^{-\left(l^{2}+m^{2}+n^{2}\right)\left(t-t_{1}\right)}$, and thus will be majorized by

$$
\left\|F\left(u_{n}\right)-F\left(u_{n-1}\right)\right\| e^{-\left(l^{2}+m^{2}+n^{2}\right)\left(t-t_{1}\right)} .
$$

Since $F\left(u_{n}\right)-F\left(u_{n-1}\right)=F^{\prime}(\xi)\left(u_{n}-u_{n-1}\right)$, where $\|\xi\| \leqq 2 d_{1}\|f\|$, we have

(8) Unfortunately $n$ has been used in two different senses in this and some later formulas, once associated with the triple $(l, m, n)$, and once as the letter signifying the $n$th approximation. No confusion seems to arise from this, however. 


$$
\left\|F\left(u_{n}\right)-F\left(u_{n-1}\right)\right\| \leqq\left\|F^{\prime}(\xi)\right\|\left\|u_{n}-u_{n-1}\right\| \leqq c_{2} d_{1}\|f\|\left\|u_{n}-u_{n-1}\right\|,
$$

provided $\|f\|$ is sufficiently small. Thus,

$$
\left\|\int_{0}^{t-d}\right\| \leqq c_{2} d_{1}\|f\|\left\|u_{n}-u_{n-1}\right\| \sum_{l, m, n=1}^{\infty} \frac{e^{-\left(l^{2}+m^{2}+n^{2}\right) d}}{l^{2}+m^{2}+n^{2}} .
$$

Similarly, in $(t-d, t)$, since we are now dealing with an expression formed from the Fourier series of $F\left(u_{n}\right)-F\left(u_{n-1}\right)$, we have, using Lemma 1,

$$
\left\|\int_{t-d}^{t}\right\| \leqq d_{1} d\left\|F\left(u_{n}\right)-F\left(u_{n-1}\right)\right\| \leqq 8 d_{1}^{2} d c_{2}\|f\|\left\|u_{n}-u_{n-1}\right\| .
$$

Thus, finally,

$$
\begin{aligned}
\left\|u_{n+1}-u_{n}\right\| \leqq & \left\|\int_{0}^{t-d}\right\|+\left\|\int_{t-d}^{t}\right\| \leqq 8 d_{1}^{2} d c_{2}\|f\|\left\|u_{n}-u_{n-1}\right\| \\
& +8 c_{2} d_{1}\|f\|\left\|u_{n}-u_{n-1}\right\| \sum_{l, m, n=1}^{\infty} \frac{e^{-\left(l^{2}+m^{2}+n^{2}\right) d}}{l^{2}+m^{2}+n^{2}} .
\end{aligned}
$$

Choose $d$ so that

$$
d d_{1}=\sum_{l, m, n=1}^{\infty} \frac{e^{-\left(l^{2}+m+n^{2}\right) d}}{l^{2}+m^{2}+n^{2}}
$$

and then (4.13) becomes

$$
\left\|u_{n+1}-u_{n}\right\| \leqq 16 d_{1}^{2} d c_{2}\|f\|\left\|u_{n}-u_{n-1}\right\|,
$$

whence if $\|f\|$ is sufficiently small, we have convergence of $\sum_{n=0}^{\infty}\left\|u_{n+1}-u_{n}\right\|$, and thus uniform convergence of $\sum_{n=0}^{\infty}\left(u_{n+1}-u_{n}\right)$. Thus $u_{n}$ converges uniformly to a function $u$ which satisfies the integral equation, since

$$
u_{n+1}=u_{0}-T\left(u_{n}\right)
$$

implies

$$
u=\lim _{n \rightarrow \infty} u_{n+1}=u_{0}-\lim _{n \rightarrow \infty} T\left(u_{n}\right)=u_{0}-T(u) .
$$

Whereas, in the theory of ordinary differential equations we would now be finished with the proof, since it would be immediately obvious that $u$ satisfied the differential equation from which the integral equation was derived, at this point in our proof we have no surety that $u$ actually possesses partial derivatives. The proof is now complicated by the necessity of proving this fact. It is this complication which seems to prevent the application of the fixed-point method to our problem. We can weaken the hypothesis, as we did in proving the stability result, and obtain the existence of a solution of the 
integral equation, which may be considered as a generalized solution of the partial differential equation, but it does not seem possible to obtain the existence of a solution of the partial differential equation this way without imposing restrictions on $f(x, y, z)$, which are very severe.

This point brings up some interesting questions. Since we have proved that $u_{n}$ converges uniformly to $u$, we have a sequence, $u_{n}$, with the property

$$
\begin{aligned}
u_{n} & \rightarrow u, \\
\left(u_{n}\right)_{x x}+\left(u_{n}\right)_{y y}+\left(u_{n}\right)_{z z}-\left(u_{n}\right)_{t} & \rightarrow F(u),
\end{aligned}
$$

since the recurrence relations of (4.2) are equivalent to the equations

$$
\left(u_{n+1}\right)_{x x}+\left(u_{n+1}\right)_{y y}+\left(u_{n+1}\right)_{z z}-\left(u_{n+1}\right)_{t}=F\left(u_{n}\right), \quad n \geqq 0
$$

(this will be proven subsequently).

An interesting question, apart from its origin, is whether or not from (4.18) it follows that

$$
u_{x x}+u_{y y}+u_{z z}-u_{t}=F(u) .
$$

Questions of this type have been discussed by Friedrichs [3] for other types of operators.

For the case of the operators of ordinary differential equations, it can be answered in the affirmative if the operator is linear, and satisfies some other light conditions. The question seems to be of independent interest in the general case, and we hope to return to this question.

We now proceed to show that (4.20) is valid in our case. It seems difficult to give a proof which covers the entire $t$-range at one time, and what we shall do is confine ourselves to a certain finite $t$-interval $\left(0, t_{2}\right)$, where $t_{2}$ will be defined below, prove the result for this interval, and show that the conditions which permitted the proof over the interval $\left(0, t_{2}\right)$ are duplicated over $\left(t_{2}, 2 t_{2}\right)$, and thus the $t$-interval can be extended to cover the entire interval $(0, \infty)$.

What we shall show below is that for any $t_{2}>0$, provided $\|f\|$ is sufficiently small, depending upon $t_{2}$, the functions $\left(u_{n}\right)_{x x},\left(u_{n}\right)_{y y},\left(u_{n}\right)_{z z}$ are equi-continuous in any closed sub-interval of $\left(0, t_{2}\right)$. This result will allow us to use a selection process, which will yield (4.20) over this finite $t$-interval. We now consider the new boundary value problem, where $t$ begins with the value $t_{2}$, and $u$ satisfies the differential equation of (1.14), the first boundary condition of (1.15) and the condition

$$
\lim _{t \rightarrow t_{2}+0} u=\lim _{t \rightarrow t_{2}-0} u,
$$

where the $u$ appearing in the right-hand side is one obtained above over the finite interval. The same method will permit us to extend the interval of validity of $(4.20)$ to $\left(0,2 t_{2}\right)$, if $u\left(t_{2}\right)$ is small enough. We now show that if $t_{2}$ 
is suitably chosen this will be so. We have

$$
u\left(t_{2}\right)=u_{0}\left(t_{2}\right)-\int_{0}^{t_{2}}\left[\sum_{l, m, n=1}^{\infty} F_{m n}\left(t_{1}\right) e^{-\left(l^{2}+m^{2}+n^{2}\right)\left(t_{2}-t_{1}\right)} \psi_{l m n}\right] d t_{1} .
$$

Thus, applying Lemma 1, we have

$$
\left\|u\left(t_{2}\right)\right\| \leqq\|f\| \sum_{l, m, n=1}^{\infty} e^{-\left(l^{2}+m^{2}+n^{2}\right) t_{2}}+t_{2}\|F(u)\| .
$$

Since $F(u)=o(\|u\|)$ as $\|u\| \rightarrow 0$, and $\|u\| \leqq 2 d_{1}\|f\|$, we have,

$$
\left\|u\left(t_{2}\right)\right\| \leqq\|f\| \sum_{l, m, n=1}^{\infty} e^{-\left(l^{2}+m^{2}+n^{2}\right) t_{2}}+c_{1} t_{2}\|f\|,
$$

where $c_{1}$ can be made arbitrarily small by choosing $\|f\|$ small enough. Now

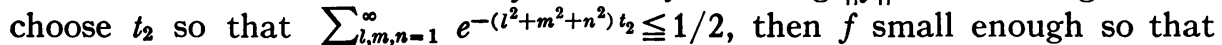
$c_{1} t_{2} \leqq 1 / 2$, then $\left\|u\left(t_{2}\right)\right\| \leqq\|f\|$, and the previous conditions are duplicated. All conditions require that $\|f\|$ be small enough; thus there are no contradictions.

In treating the interval $\left(0, t_{2}\right)$, we shall be forced to use a selection theorem, since it does not seem possible to prove that $\left(u_{n}\right)_{x x}$ converges uniformly in any $t$-interval not including 0 by the above methods. What we shall show is that the $\left(u_{n}\right)_{x x},\left(u_{n}\right)_{y y},\left(u_{n}\right)_{z z}$ are uniformly bounded for $x, y, z$ in $R$, by a function of $t$, which, as to be expected, $\rightarrow \infty$ as $t \rightarrow 0$. However, restricting ourselves to any fixed $t$-interval, not including the origin, once we have proved equicontinuity, we can use the Arzela selection theorem. The use of the diagonal process will then allow us to have convergence over the interval $\left(0<t \leqq t_{2}\right)$, and uniformly over any closed sub-interval.

We shall confine our attention to $\left(u_{n}\right)_{x}$ and $\left(u_{n}\right)_{x x}$. Similar bounds will hold for the derivatives with respect to $y, z$ and $t$.

We begin with $u_{0}$. For any $t>0$, we have

$$
\max _{R}\left|\left(u_{0}\right)_{x}\right| \leqq \sum_{l, m, n=1}^{\infty} l e^{-\left(l^{2}+m^{2}+n^{2}\right) t}=\left(\sum_{l=1}^{\infty} l e^{-l^{2} t}\right) \cdot\left(\sum_{l=1}^{\infty} e^{-l^{2} t}\right)^{2} .
$$

Let us assume that we have shown that

$$
\max _{R}\left|\left(u_{n}\right)_{x}\right| \leqq 2 \sum_{l, m, n=1}^{\infty} l e^{-\left(l^{2}+m^{2}+n^{2}\right) t}, \quad t>0, N=0,1, \cdots, n .
$$

We shall show that this result also holds for $(n+1)$, provided $\|f\|$ is sufficiently small. Since we have the result for $n=0$, the induction can be started. We have

$$
\left(u_{n+1}\right)_{x}=\left(u_{0}\right)_{x}-\int_{0}^{t}\left[\sum_{l, m, n=1}^{\infty} F_{l m n}\left(u_{n}\left(t_{1}\right), t_{1}\right) e^{-\left(l^{2}+m^{2}+n^{2}\right)\left(t-t_{1}\right)}\left(\psi_{l m n}\right)_{x}\right] d t_{1}
$$


However, it is necessary to justify the term-by-term differentiation. Over any interval $(0, t-d), 0<d<t$, it is legitimate because of the absolute convergence of the differentiated series, while over the interval $(t-d, t)$ it holds because of the uniform convergence of the series for $F\left(u_{n}\right)_{x}$. Since $u_{0}$ has derivatives of all orders with respect to $x, y, z$, for $t>0$, it follows by iteration of the recurrence relations that these properties persist for all $n \geqq 0$.

It is now convenient to consider $F(u)$ as an odd function of $x, y, z$, defined over intervals $-\pi \leqq x \leqq 0,-\pi \leqq y \leqq 0$, by this property. Since $F(u)$ is zero at $x-0, y=0, z=0$, for $t>0$, there is no discontinuity, except perhaps for $t=0$, which need not bother us, since we shall use this extended representation only for $t>0$. All the derivatives will also exist at $x=0, y=0, z=0$.

Thus the series under the integral sign in (4.27) will represent the, Fourier series of $F\left(u_{n}\right)_{x}$ over the region $R$, with the convergence factors $e^{-\left(l^{2}+m^{2}+n^{2}\right)\left(t-t_{1}\right)}$ appearing in each term.

We now split the interval into two parts, $(0, t / 2),(t / 2, t)$. In the interval $(0, t / 2)$, each term in the series of the integrand is less than $\left\|F\left(u_{n}\right)\right\|$, multiplied by $l e^{-\left(l^{2}+m^{2}+n^{2}\right)\left(t-t_{1}\right)}$, and thus we have

$$
\max _{R}\left|\int_{0}^{t / 2}\right| \leqq\left\|F\left(u_{n}\right)\right\| \sum_{l, m, n=1}^{\infty} \frac{l e^{-\left(l^{2}+m^{2}+n^{2}\right) t / 2}}{l^{2}+m^{2}+n^{2}} .
$$

In the interval $(t / 2, t)$, we use the fact that the series appearing is the Fourier series for the function $F\left(u_{n}\right)_{x}=F^{\prime}\left(u_{n}\right)\left(u_{n}\right)_{x}$, with the convergence factors $e^{-\left(l^{2}+m^{2}+n^{2}\right)\left(t-t_{1}\right)}$. Thus, using Lemma 1 ,

$$
\max _{R}\left|\int_{t / 2}^{t}\right| \leqq d_{1}\left\|F^{\prime}\left(u_{n}\right)\right\| \frac{t}{2} \sum_{l, m, n=1}^{\infty} l e^{-\left(l^{2}+m^{2}+n^{2}\right) t / 2} .
$$

Since we are considering only a finite interval $(0, t / 2)$, we have in $(0, t / 2)$

$$
\sum_{l, m, n=1}^{\infty} l e^{-\left(l^{2}+m^{2}+n^{2}\right) t / 2} \leqq c\left(t_{2}\right) \sum_{l, m, n=1}^{\infty} l e^{-\left(l^{2}+m^{2}+n^{2}\right) t},
$$

since, fortunately, the asymptotic value of the right-hand side of (4.25) as $t \rightarrow 0$ is $c / t^{2}$, and thus only differs from the asymptotic value of the left-hand side of (4.30) by a multiplicative constant. Thus

$$
\begin{aligned}
\max _{R}\left|\left(u_{n+1}\right)_{x}\right| & \leqq \max _{R}\left|\left(u_{0}\right)_{x}\right|+\max _{R}\left|\int_{0}^{t / 2}\right|+\max _{R}\left|\int_{t / 2}^{t}\right| \\
& \leqq\left[\sum_{l, m, n=1}^{\infty} l e^{-\left(l^{2}+m^{2}+n^{2}\right) t}\right]\left[1+\left\|F\left(u_{n}\right)\right\| c(t / 2)\right. \\
& \left.\quad+d_{1} t_{2}\left\|F^{\prime}\left(u_{n}\right)\right\| c(t / 2)\right] \\
& \leqq 2 \sum_{l, m, n=1}^{\infty} l e^{-\left(l^{2}+m^{2}+n^{2}\right) t}
\end{aligned}
$$


provided $\|f\|$ is small enough. This follows from the inequalities

$$
\left\|F\left(u_{n}\right)\right\|=o\left(\left\|u_{n}\right\|\right)=o(\|f\|),\left\|F^{\prime}\left(u_{n}\right)\right\|=O\left(\left\|u_{n}\right\|\right)=O(\|f\|) .
$$

Since $c(t / 2)$ increases as $t / 2$ increases, and a decrease in $\|f\|$ causes a decrease in the required $t / 2$, we can always satisfy all conditions by taking $\|f\|$ small enough.

The same method is used to obtain a bound for $\left(u_{n}\right)_{x x}$, using the relation

$$
F(u)_{x x}=F^{\prime \prime}(u) u_{x}^{2}+F^{\prime}(u) u_{x x} .
$$

Arguing very crudely, we obtain an estimate $\max _{R}\left|\left(u_{n}\right)_{x x}\right| \leqq c / t^{5 / 2}$. Continuing, we can find uniform bounds for $\left(u_{n}\right)_{x x x}$, and similarly for all partial derivatives.

Our estimates show that if we fix $t>0$, then the sequences, $\left(u_{n}\right)_{x},\left(u_{n}\right)_{x x}$ are uniformly bounded over $R$, and because of the bounds on the other partial derivatives, equi-continuous functions of $x, y, z$ over the finite region $R$.

We can now use the Arzela selection theorem for the region $R$, with $t$ fixed. Thus there exists a subsequence $\left(n_{k}\right)$ such that

$$
\left(u_{n_{k}}\right)_{x x} \rightarrow v_{1},\left(u_{n_{k}}\right)_{x} \rightarrow W \quad(\rightarrow \text { denotes uniform convergence). }
$$

Now since $u=0$ at $x=0, t>0$, if $\left(u_{n_{k}}\right)_{x} \rightarrow W, u_{n_{k}} \rightarrow u$, then $W=u_{x}$. This follows from the integral relation

$$
u_{n_{k}}=\int_{0}^{x}\left(u_{n_{k}}\right)_{x} d x
$$

together with the uniform convergence. Since $u_{x}=0$ at $x=\pi / 2$, also $v=W_{x}$ $=u_{x x}$.

Since

$$
\left(u_{n}\right)_{t}=F\left(u_{n-1}\right)-\left(u_{n}\right)_{x x}-\left(u_{n}\right)_{y y}-\left(u_{n}\right)_{z z},
$$

and $u_{n} \rightarrow u$, as $n \rightarrow \infty$, through any sequence, if we let $n \rightarrow \infty$ through the sequence $n_{k}$, we see that $\left(u_{n_{k}}\right)_{t}$ converges uniformly and

$$
\lim _{n_{k \rightarrow \infty}}\left(u_{n_{k}}\right)_{t}=F(u)-u_{x x}-u_{y y}-u_{z z}=r .
$$

We now have to investigate whether $r=u_{t}$, as we suspect. We could use the bounded convergence of $u_{n_{k}}$ and integrate between $t$ and $t_{2}, t>0$, to show that $r=u_{t}$ almost everywhere, but this is unsatisfactory, and it takes only an additional step to show that we can choose a subsequence of $n_{k}$ such that $u_{n_{k}} \rightarrow r$, with respect to $t_{1}$ in any closed interval $\left(t, t_{2}\right), t>0$. To do that we have merely to verify that $\left(u_{n}\right)_{t t}$ is uniformly bounded. But since

$$
\left(u_{n}\right)_{t}=\left(u_{n}\right)_{x x}+\left(u_{n}\right)_{y y}+\left(u_{n}\right)_{z z}-F\left(u_{n-1}\right), \quad n \geqq 1,
$$

it follows that $\left(u_{n}\right)_{t t}$ can be expressed again in terms of bounds on $\left(u_{n}\right)_{x x x x}$, 
and so on. We could also obtain such bounds directly from the recurrence relations. Thus, in any fixed region, $R \times\left(t, t_{2}\right), t>0,\left(u_{n_{k}}\right)_{t} \rightarrow r$, and thus $r=u_{t}$.

Thus the function $u$ satisfies the partial differential equation.

We can repeat the proof which showed uniform boundedness of $\left\|u_{n}\right\|$, to show that $\left|u_{n}\right| \leqq d_{2} e^{-t / 2}$ as $t \rightarrow \infty$, where $d_{2}$ is a constant determined by the initial conditions. This will show that $u \rightarrow 0$ as $t \rightarrow \infty$. This estimate can be improved to $O\left(e^{-3 t}\right)$ easily.

The proof is now complete as far as concerns the demonstration of the existence of a solution of the equation, satisfying the boundary conditions and approaching 0 as $t \rightarrow \infty$.

The existence of the one-dimensional functional manifold has also been shown. This manifold of solutions is defined by the "sphere" of all functions $f(x, y, z)$ for which $\|f\|$ is sufficiently small.

We now come to the proof of uniqueness for functions belonging to $S$. As we have shown in Lemma 2, any solution, $U$, belonging to $S$, is a solution of the integral equation

$$
U=u_{0}-T(U) .
$$

To prove uniqueness if $\|f\|$ is sufficiently small, we now use the method used in ordinary differential equations. We have

$$
\begin{aligned}
& \max _{R}\left|U-u_{0}\right| \leqq \max _{R}|T(U)|, \\
& \max _{R}\left|U-u_{n+1}\right| \leqq \max _{R}\left|T(U)-T\left(u_{n}\right)\right|, \quad n \geqq 0 .
\end{aligned}
$$

What we shall do is iterate these relations and show that $\max _{R}\left|U-u_{n}\right|$ $\rightarrow 0$ as $n \rightarrow \infty$, whence $U \equiv u$.

As we showed in Theorem 2, $\|U\| \leqq 2 d_{1}\|f\|$, provided $\|f\|$ is small enough. Thus, using Lemma 1,

$$
\max _{R}\left|U-u_{0}\right| \leqq 2 d_{1}^{2}\|f\| t
$$

Using this inequality in the inequality

$$
\max _{R}\left|U-u_{1}\right| \leqq \max _{R}\left|T(U)-T\left(u_{0}\right)\right|,
$$

after we have applied the mean-value theorem to reduce $\left|T(U)-T\left(u_{0}\right)\right|$, as in (4.10), we obtain, applying Lemma 1 again

$$
\max _{R}\left|U-u_{1}\right| \leqq 2 d_{1}^{2}\|f\|\left(d_{1} F^{\prime}\left(2 d_{1}\|f\|\right)\right) \frac{t^{2}}{2} .
$$

This is satisfactory provided $F^{\prime}\left(2 d_{1}\|f\|\right)<\infty$, which it must be, as we can see by returning to Theorem 2 and observing how the proof went.

It is now easy to show by induction that 


$$
\max _{R}\left|U-u_{n}\right| \leqq 2 d_{1}^{2}\|f\|\left(d_{1} F^{\prime}\left(2 d_{1}\|f\|\right)^{n}\right) \frac{t^{n+1}}{(n+1) !} .
$$

Thus $\max _{R}\left|U-u_{n}\right| \rightarrow 0$ as $n \rightarrow \infty$, whence $U \equiv u$.

5. The equations of Sturm-Liouville type. Let us begin by discussing the one-dimensional case

$$
u_{x x}-u_{t}-q(x) u=F(u, x, t)
$$

with the boundary conditions

$$
\begin{aligned}
u & =0 & \text { at } x=0, \pi, t>0, \\
\lim _{t \rightarrow 0} u & =f(x) . &
\end{aligned}
$$

Let us consider the linear equation obtained by setting $F=0$. We use the standard separation of variables method, and set $u(x, t)=u(x) e^{-\lambda^{2} t}$. In this way we arrive at the classical Sturm-Liouville equation

$$
u^{\prime \prime}+\left[\lambda^{2}+q(x)\right] u=0, \quad u(0)=u(\pi)=0 .
$$

To obtain the solution of (5.1), with $F=0$, we must find the eigenfunctions of (5.3), $u_{n}(x)$, corresponding to the eigenvalues, $\lambda_{n}$, and then consider

$$
u=\sum_{n=1}^{\infty} a_{n} u_{n}(x) e^{-\lambda_{n}^{2} t},
$$

where

$$
u \sim \sum_{n=1}^{\infty} a_{n} u_{n}(x)
$$

If $q(x)$ is a continuous function over the interval $(0, \pi)$, we know that the asymptotic forms of the $\lambda_{n}$ and the normalized eigenfunctions, $u_{n}$, are, [6],

$$
\lambda_{n}=n+1+O\left(n^{-1}\right), \quad u_{n}(x)=(2 / \pi) \sin (n+1) x+O\left(n^{-1}\right) .
$$

Thus we would expect the analogue of Lemma 1 to be true here, and using the equi-summability theorem of Haar [6] we have the following result:

LEMMA 3. If

$$
u \sim \sum_{n=1}^{\infty} a_{n} u_{n}
$$

then

$$
\left|\sum_{n=1}^{\infty} a_{n} u_{n} e^{-n^{2} t}\right| \leqq d_{3}\|u\|
$$


where $\|u\|=\max _{0 \leqq x \leqq \pi}|u(x)|,\|u(x, t)\|=\max _{R}|u(x, t)|$, where $R=(0 \leqq x \leqq \pi)$ $X(0 \leqq t<\infty)$.

Lemma 3 is proved using the method of Fejer, the same method used by Eversull [3] in the three-dimensional case.

Once we have established Lemma 2 , there is no difficulty obtaining results corresponding to Theorem 1.

For reasons we shall discuss below, we do not wish to dwell upon this topic to any length at the present moment.

If we consider the more general equation

$$
u_{x x}+u_{y y}+u_{z z}-u_{t}+q(x, y, z) u=0
$$

where

$$
u=0 \quad \text { on } \quad B, \quad t>0, \quad \lim _{t \rightarrow 0} u=f(x, y, z),
$$

we obtain the corresponding eigenvalue problem

$$
u_{x x}+u_{y y}+u_{z z}+\left[\lambda^{2}+q(x, y, z)\right] u=0, \quad u=0 \quad \text { on } \quad B .
$$

Various precise results are known concerning the distribution of the eigenvalues of (5.11), but there does not seem to be much information concerning the asymptotic behavior of the eigenfunctions. Most probably, for the boundary conditions of (1.3), we have that

$$
\lambda_{l m n} \sim\left(l^{2}+m^{2}+n^{2}\right)^{1 / 2}, \quad u_{l m n} \sim\left(\left(\frac{2}{\pi}\right)^{1 / 2}\right)^{3} \sin l x \sin m y \sin n z,
$$

and corresponding equi-summability theorems.

The result we really desire is the analogue of Lemma 1, namely that

$$
\left\|\sum a_{l m n} u_{l m n} e^{-\lambda^{2} l m n t}\right\| \leqq d_{4}\|u\| \text {, }
$$

if

$$
u \sim \sum_{l, m, n=1}^{\infty} a_{l m n} u_{l m n} .
$$

This problem seems to be worthy of attention apart from its application to the results given in this paper.

An investigation of the summability of double Sturm-Liouville series has recently been given by Mitchell [14].

Lemma 3 and the hypothesized result of (5.13) are connected with some ideas in the theory of operators which we think interesting enough to mention here. Considering the equation (5.1) with the boundary conditions (5.2), we see that $u$ is a functional of $f, u=T(f)$. If $F$ is 0 , it is an additive functional, $T\left(f_{1}+f_{2}\right)=T\left(f_{1}\right)+T\left(f_{2}\right)$. Furthermore, Lemma 3 states the important result 
that $u$ is a continuous functional, since boundedness plus additivity yield continuity. Thus, in the sense of Banach, $u$ is a linear operator defined over the space of continuous functions.

The hoped for result stated in (5.13) implies that the operator $T(f)$, defined by (5.11), is also a linear operator. Theorem 2 shows that the solution of its partial differential equation and boundary conditions, while not linear, is nevertheless continuous, as long as $f$ is sufficiently small.

It would be desirable to have a method of proof that would yield these results, without having to examine the finer structure of the eigenvalues and eigenfunctions. It may be expected, however, that each type of equation and region will require separate proofs. We shall discuss below some types of equations that can be treated immediately, and some results for other types of regions.

6. Other types of partial differential equations. The same method used by Eversull [1] to prove Lemma 1 furnishes proofs of the following:

LEMMA 4. If

$$
u \sim \sum_{l, m, n=1}^{\infty} a_{l m n} \sin l x \sin m y \sin n z=\sum_{l, m, n=1}^{\infty} a_{l m n} \psi_{l m n}
$$

then

$$
\begin{aligned}
& \left\|\sum_{l, m, n=1}^{\infty} a_{l m n} \psi_{l m n} e^{-\left(l^{2}+m^{2}+n^{2}\right)^{2} t}\right\| \leqq d_{5}\|u\|, \\
& \left\|\sum_{l, m, n=1}^{\infty} a_{l m n} \psi_{l m n} e^{-\left(l 4+m^{4}+n^{4}\right) t}\right\| \leqq d_{6}\|u\| .
\end{aligned}
$$

Thus the following equations may be discussed, and results analogous to Theorems 1 and 2 obtained:

$$
\begin{aligned}
\nabla^{2} u-u_{t} & =F(u, x, y, z, t), \\
u_{x x x x}+u_{y y y y}+u_{z z z z}-u_{t} & =F(u, x, y, z, t) .
\end{aligned}
$$

7. Other type of boundary conditions. Siddiqi [12] has given a method based upon change of variables for handling various types of boundary conditions. These transformations of variables reduce the problem to that of (1.1) with boundary conditions (1.3), for the one-dimensional case.

We wish to consider now an essentially different type of region than the cube, namely the semi-infinite circular cylinder.

If we consider, Moore [8], the problem of determining the steady flow of heat in a semi-infinite cylinder whose base is kept at a temperature $v=f(r)$, where $r$ is the perpendicular listance from the axis of the cylinder, we have the equation 


$$
\frac{1}{r} \frac{\partial}{\partial r}\left(r \frac{\partial v}{\partial r}\right)+\frac{\partial^{2} v}{\partial r^{2}}=F(v, r, z), \quad z>0,0<r<1,
$$

where $F$ is a nonlinear term in $v$, with the boundary conditions

$$
\begin{aligned}
\frac{\partial v}{\partial r}+h v & =0, & v>0, r=1, \\
\lim _{s \rightarrow 0} v & =f(r) . &
\end{aligned}
$$

If we consider the linear problem, $F=0$, first, it is easily shown that $e^{-\lambda z} J_{0}(\lambda r)$ is a solution of (7.1) satisfying the first condition of (7.2), provided $\lambda$ is a root of

$$
l \lambda J_{0}^{\prime}(\lambda)+h J_{0}(\lambda)=0 .
$$

Thus a solution of the linear case is afforded by

$$
v=\sum_{k=1}^{\infty} a_{k} J_{0}\left(\lambda_{k} r\right) e^{-\lambda_{k} z}
$$

where

$$
f(r) \sim \sum_{k=1}^{\infty} a_{k} J_{0}\left(\lambda_{k} r\right)
$$

Moore now shows that

$$
\left\|r^{1 / 2} v\right\| \leqq d_{6}\left\|r^{1 / 2} f\right\|
$$

where $\left\|r^{1 / 2} f\right\|=\max _{0 \leqq r \leqq 1}\left|r^{1 / 2} f\right|,\left\|r^{1 / 2} v\right\|=\max _{R}\left|r^{1 / 2} v\right|$, where $R=(0 \leqq r \leqq 1)$ $X(0 \leqq z<\infty)$.

With this as a start, we can now proceed to prove results corresponding to Theorems 1 and 2.

Since the results for many other types of regions such as spheres, ellipsoids, and so on, are still unknown, we prefer to wait until we can state results for these regions too, and discuss the cylindrical region completely at that time.

8. Application of the method to nonlinear integro-differential equations. Let us consider the nonlinear integro-differential equation

$$
\begin{aligned}
\frac{d y}{d z}=a_{0}(u, z) & +\int_{0}^{1} y\left(z, \lambda_{1}\right) a_{1}\left(\lambda_{1}, u\right) d \lambda_{1} \\
& +\int_{0}^{1} \int_{0}^{1}\left[y\left(z, \lambda_{1}\right) y\left(z, \lambda_{2}\right) a_{2}\left(\lambda_{1}, \lambda_{2}, u\right)\right] d \lambda_{1} d \lambda_{2}
\end{aligned}
$$

with the boundary condition 


$$
\lim _{z \rightarrow 0} y=f(u)\left({ }^{9}\right) .
$$

Linear integro-differential equations of this type have been considered by Schlesinger [9], and Hilb [5]. We shall now sketch a method of treating equations of this type, based upon the previous ideas.

Consider the equation

$$
\frac{d y}{d z}=A(u, z)+\int_{0}^{1} y\left(z, \lambda_{1}\right) a_{1}\left(\lambda_{1}, u\right) d \lambda_{1} .
$$

Let us try a solution of the form $y=\sum_{n=1}^{\infty} \psi_{n}(z) \phi_{n}(u)$, where $\phi_{n}(u)$ is a member of the orthonormal sequence of eigenfunctions of $a_{1}\left(\lambda_{1}, u\right)$, that is

$$
\lambda_{n} \phi_{n}(u)=\int_{0}^{1} \phi_{n}\left(\lambda_{1}\right) a_{1}\left(\lambda_{1}, u\right) d \lambda_{1}, \quad \int_{0}^{1} \phi_{n}(u) \phi_{m}(u) d u=\delta(m, n) .
$$

Let

$$
A(u, z) \sim \sum_{n=1}^{\infty} A_{n}(z) \phi_{n}(u) .
$$

Then equating coefficients after substituting into (8.3), we obtain the equations

$$
\psi_{n}^{\prime}(z)=A_{n}(z)+\lambda_{n} \psi_{n}(z)
$$

If $f(u) \sim \sum_{n=1}^{\infty} f_{n} \phi_{n}(u)$, we use the boundary condition $\psi_{n}(0)=f_{n}$, and obtain the following solution of (8.6)

$$
\psi_{n}(z)=f_{n} e^{\lambda_{n} z}+\int_{0}^{z} e^{n\left(-\sigma_{1}\right)} A_{n}\left(z_{1}\right) d z_{1}
$$

and thus the formal solution

$$
y=\sum_{n=1}^{\infty} f_{n} e^{\lambda_{n} z} \phi_{n}(v)+\sum_{n=1}^{\infty}\left\{\int_{0}^{z} e^{\lambda_{n}\left(z-z_{1}\right)} A_{n}\left(z_{1}\right) \phi_{n}(v) d z_{1}\right\}
$$

of (8.3).

This expression is similar to that of (2.5), and we may now use it as we did in Theorem 1 . We would require that $\lambda_{n}<0$, and that

$$
\left|\sum_{n=1}^{\infty} f_{n} e^{\lambda_{n} z} \phi_{n}(u)\right| \leqq d_{0}\|u\| .
$$

Instead of the integro-differential equation of (8.1), we could consider

(9) $a_{1}(x, y)$ is assumed to be symmetric in $x$ and $y$. 


$$
\frac{\partial y}{\partial z}=a_{0}(u, z)+\int_{0}^{z} a_{1}(z, u)\left[y\left(z, \lambda_{1}\right)+F(y)\right] d \lambda_{1},
$$

where $F$ is a nonlinear function of $y$.

\section{REFERENCES}

1. R. Bellman, On the stability of systems of differential equations, Proc. Nat. Acad. Sci. U.S.A. vol. 32 (1946) pp. 190-193.

2. B. M. Eversull, On convergence factors in triple series and the triple Fourier's series, Ann. of Math. vol. 24 (1923) pp. 141-165.

3. K. O. Friedrichs, The identity of weak and strong extensions of differential operators, Trans. Amer. Math. Soc. vol. 55 (1944) pp. 132-151.

4. M. Gevrey, Sur les equations aux dérivées partielles du type parabolique, Journal de Mathématiques (6) vol. 10 (1914) pp. 105-149.

5. O. Hilb, Zur Theorie der linearen Integrodifferentialgleichungen, Math. Ann. vol. 77 (1916) pp. 514-535.

6. E. L. Ince, Ordinary differential equations, New York, 1944, pp. 272-277.

7. S. Minakshisundaram, Fourier Ansatz and non-linear parabolic equations, J. Indian Math. Soc. N.S. vol. 7 (1943) pp. 129-143.

8. C. N. Moore, The summability of the developments in Bessel functions, with applications, Trans. Amer. Math. Soc. vol. 10 (1909) pp. 391-435.

9. L. Schlesinger, Zur Theorie du linearen Integrodifferentialgleichungen, Jber. Deutschen. Math. Verein (1915) pp. 84-123.

10. R. Siddiqi, On the equation of heat conduction in wave mechanics, J. Indian Math. Soc. vol. 20 (1933) pp. 226-235.

11. - Boundary problems in non-linear parabolic equations, J. Indian Math. Soc. N.S. vol. 1 (1934-1935).

12. - Zur Theorie du nichtlinearen partiellen Differentialgleichungen vom parabolischen Typus, Math. Zeit. vol. 35 (1932) pp. 464-484. 636.

13. J. Mitchell, On double Sturm-Liouville series, Amer. J. Math. vol. 45 (1943) pp. 616-

See also

$\mathrm{R}$. Bellman, On the boundedness of solutions of nonlinear differential and difference equations, Trans. Amer. Math. Soc. vol. 62 (1947) pp. 357-386.

Princeton University,

Princeton, N. J. 